\title{
Outbreaks of Foodborne Illness Associated with Common Berries, 1983 through $2019^{1}$
}

\author{
Matthew D. Krug, Mary Palumbo, Linda J. Harris, and Michelle D. Danyluk²
}

\section{Introduction}

Fresh and frozen common berries (i.e., blackberries, blueberries, raspberries, and strawberries) are popular and healthy foods. When berries are picked for fresh consumption, they are either placed directly in retail containers in the field or packed in a packinghouse without washing because they are highly perishable. Berries may be washed before freezing, but they are not usually blanched or heattreated unless they are used in preserves or other processed products. Thus, there is typically no "kill step" that would eliminate pathogens in fresh or frozen berries.

Berries may be served mixed with other foods, such as in salads or desserts, and these foods may contain more than one kind of berry or other fruit. Epidemiologists have more difficulty accurately determining the food vehicle during a foodborne illness outbreak when the outbreak is associated with mixed foods, such as mixed berries. The viral and parasitic pathogens that have caused outbreaks associated with consumption of berries are difficult to detect in foods. The laboratory methods used to detect these pathogens have only recently been developed or are still under development. In November 2018, FDA began a 2-year survey of frozen berries (strawberries, raspberries, and blackberries) for the presence of norovirus and hepatitis A.
As of September 30, 2019, 812 samples (339 domestic and 473 imported) had been screened. Of the frozen berries sampled, genetic material from hepatitis A virus was found in five samples (three domestic: one strawberry, one raspberry, and one blackberry; two imported: two raspberry) and genetic material from norovirus in eight samples (three domestic: one strawberry, two raspberry; five imported: two strawberry, one raspberry, two blackberry). At the time of publication, testing is ongoing; the FDA's plan is to test 2,000 samples (1,000 domestic and imported each) over 2 years.

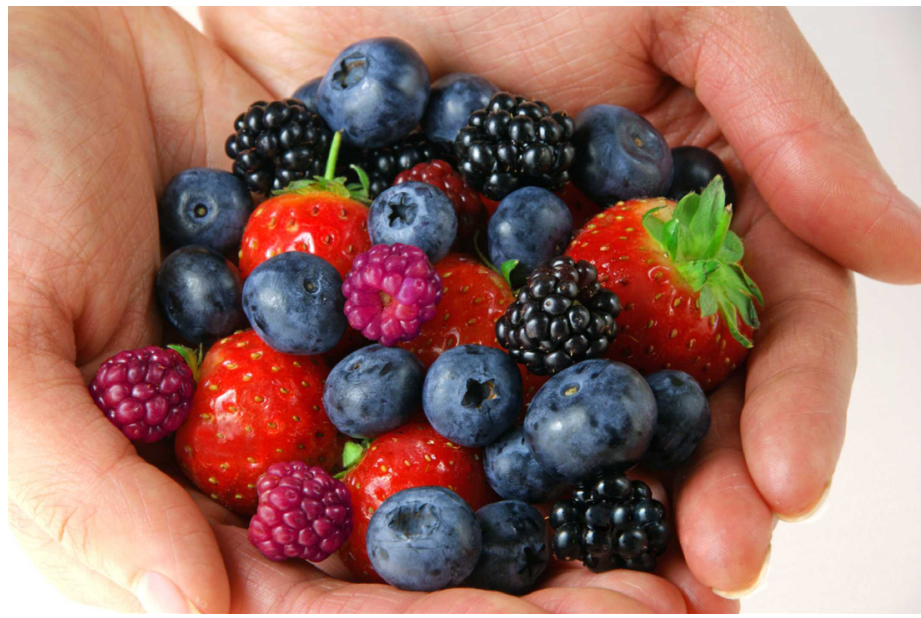

Credits: @ Peter Close (iStock)

1. This document is FSHN13-08, one of a series of the Food Science and Human Nutrition Department, UF/IFAS Extension. Original publication date November 2013. Revised June 2020. Visit the EDIS website at https://edis.ifas.ufl.edu for the currently supported version of this publication.

2. Matthew D. Krug, state specialized agent, food science, UF/IFAS Southwest Florida Research and Education Center; Mary Palumbo, retired outreach coordinator, Western Center for Food Safety, University of California, Davis; L. J. Harris, cooperative extension specialist, microbial food safety, Department of Food Science and Technology, University of California, Davis; and Michelle D. Danyluk, professor, Food Science and Human Nutrition Department, UF/IFAS Citrus REC; UF/IFAS Extension, Gainesville, FL 32611. 
This publication serves as a reference for anyone concerned about the safety of fresh and frozen berry products. Providing information for those who grow, harvest, process, transport, and serve berries to consumers is important for improving science-based food safety programs for the entire supply chain. Table 1 lists the reported outbreaks of foodborne illness from 1983 through 2019 in which specific berries or mixed berries have been identified as the food vehicle. Table 2 lists the reported outbreaks in which berries were likely the food vehicle.

\section{References}

Associated Press. 2012. "Germany: Batch of Frozen Strawberries Blamed for Outbreak of Gastroenteritis in Schools." FoxNews.com. Accessed April 2013. https://www. foxnews.com/world/2012/10/06/germany-batch-frozenstrawberries-blamed-for-outbreak-gastroenteritis-in/.

Bourquin, L. 2012. "Strawberries Implicated in Massive German Norovirus Outbreak.” Michigan State University Food Safety. Accessed April 2013. https://michiganstateuniversityfoodsafety.wordpress.com/2012/10/07/strawberriesimplicated-in-massive-german-norovirus-outbreak/.

Calder, L., G. Simmons, C. Thornley, P. Taylor, K. Pritchard, G. Greening, and J. Bishop. 2003. "An Outbreak of Hepatitis A Associated with Consumption of Raw Blueberries." Epidemiol. Infect. 131 (1): 745-751.

Canadian Food Inspection Agency (CFIA). 2012. "Health Hazard Alert - Certain Western Family Brand Pomeberry Blend berries may contain Hepatitis A virus." Accessed April 2013. https://www.canada.ca/en/news/ archive/2012/04/certain-western-family-brand-pomeberryblend-berries-may-contain-hepatitis-virus.html.

Centers for Disease Control and Prevention (CDC). 1996. "Update: Outbreaks of Cyclospora cayetanensis Infection-United States and Canada, 1996." MMWR Weekly 45 (28): 611-612. https://www.cdc.gov/mmwr/preview/ mmwrhtml/00043133.htm.

Centers for Disease Control and Prevention (CDC). 1997a. "Hepatitis A Associated with Consumption of Frozen Strawberries-Michigan, March 1997." MMWR Weekly 46 (13): 288-295. https://www.cdc.gov/mmwr/preview/ mmwrhtml/00047129.htm.

Centers for Disease Control and Prevention (CDC). 1997b. "Update: Outbreaks of Cyclosporiasis-United States, 1997." MMWR Weekly 46 (21): 461-462. https://www.cdc. gov/mmwr/preview/mmwrhtml/00047716.htm.
Centers for Disease Control and Prevention (CDC). 1997c. "Outbreak of Cyclosporiasis-Northern VirginiaWashington, D.C.-Baltimore, Maryland, Metropolitan Area." MMWR Weekly 46 (30): 689-691. https://www.cdc. gov/mmwr/preview/mmwrhtml/00048551.htm.

Centers for Disease Control and Prevention (CDC). 1998. "Outbreak of Cyclosporiasis-Ontario, Canada." MMWR Weekly 47 (38): 806-809. https://www.cdc.gov/mmwr/ preview/mmwrhtml/00055016.htm.

Centers for Disease Control and Prevention (CDC). 2013. "Multistate Outbreak of Hepatitis A Potentially Associated with a Frozen Berry Blend Food Product." Accessed June 2013. https://www.cdc.gov/hepatitis/Outbreaks/2013/A1b03-31/index.html.

Centers for Disease Control and Prevention (CDC). 2018. "National Outbreak Reporting System (NORS)." Accessed November 2019. https://wwwn.cdc.gov/norsdashboard/.

Centers for Disease Control and Prevention (CDC). 2019. “U.S. Foodborne Outbreaks of Cyclosporiasis - 2000-2017." Accessed November 2019. https://www.cdc.gov/parasites/ cyclosporiasis/outbreaks/foodborneoutbreaks.html.

Centers for Disease Control and Prevention (CDC). 2020a. "Notes from the Field: Multiple Cruise Ship Outbreaks of Norovirus Associated with Frozen Fruits and Berries United States, 2019." Accessed April 2020. https://www.cdc. gov/mmwr/volumes/69/wr/mm6916a3.htm?s_cid=mm691 6a3_e\&deliveryName=USCDC_921-DM26466.

Centers for Disease Control and Prevention (CDC). 2020b. "Outbreak of Hepatitis A Virus Infections Potentially Linked to Fresh Blackberries.” Accessed April 2020. https:// www.cdc.gov/hepatitis/outbreaks/2019/hav-berries/index. htm

Colorado Department of Public Health and Environment. 2013. "May 31: Hepatitis A Outbreak Associated with Townsend Farms Organic Antioxidant Blend Frozen Berries Purchased from Costco." Accessed June 2013. https:// www.colorado.gov/cs/Satellite? $c=$ Page\&childpagename $=C$ DPHE-Main\%2FCBONLayout\&cid=1251643026247\&page name $=\mathrm{CBONWrapper}$.

Cotterelle, B., C. Drougard, J. Rolland, M. Becamel, M. Boudon, S. Pinede, O. Traore, K. Balay, P. Pothier, and E. Espie. 2005. "Outbreak of Norovirus Infection Associated with the Consumption of Frozen Raspberries, France, March 2005.” Euro Surveill. 10 (4): E050428.1. 
DW.de. 2012. "Blame Falls on Strawberries in German Mass Food Poisoning.” DW.de website. Accessed April 2013. https://www.dw.de/blame-falls-on-strawberries-in-germanmass-food-poisoning/a-16288862-1 .

European Centre for Disease Prevention and Control (ECDC) and European Food Safety Agency (EFSA). 2013a. "Epidemiological Update: Outbreak of Hepatitis A Virus Infection in Four Nordic Countries". Accessed April 2020. https://www.ecdc.europa.eu/en/news-events/ epidemiological-update-outbreak-hepatitis-virus-infectionfour-nordic-countries

European Centre for Disease Prevention and Control (ECDC) and European Food Safety Agency (EFSA). 2013b. "Outbreak of Hepatitis A Virus Infection in Residents and Travellers to Italy." Accessed June 2013. https://www. foodsafetynews.com/files/2013/06/439e.pdf.

Falkenhorst, G., L. Krusell, M. Lisby, S. B. Madsen, B. Bottiger, and K. Molbak. 2005. "Imported Frozen Raspberries Cause a Series of Norovirus Outbreaks in Denmark, 2005." Euro Surveill. 10 (9): E050922.2.

FDA (US Food and Drug Administration). "Microbial Surveillance Sampling: FY 19-20 Frozen Berries (Strawberries, Raspberries and Blackberries).” Accessed April 2020. https://www.fda.gov/food/sampling-protect-food-supply/ microbiological-surveillance-sampling-fy-19-20-frozenberries-strawberries-raspberries-and.

Fell, G., M. Boyens, and S. Baumgarte. 2007. "Frozen Berries as a Risk Factor for Outbreaks of Norovirus Gastroenteritis. Results of an Outbreak Investigation in the Summer of 2005 in Hamburg." Bundesgesundheitsblatt Gesundheitsforschung - Gesundheitsschutz 50 (2): 230-236.

Fleming, C. A., D. Caron, J. E. Gunn, and M. A. Barry. 1998. "A Foodborne Outbreak of Cyclospora cayetanensis at a Wedding: Clinical Features and Risk Factors for Illness." Arch. Intern. Med. 158 (10): 1121-1125.

Food Safety News. 2012. "Chinese Strawberries Sickened Thousands of German Students." Accessed April 2013. https://www.foodsafetynews.com/2012/10/ german-students-got-sick-on-chinese-strawberries/.

Gillesberg Lassen, S., B. Soborg, S. E. Midgley, A. Steens, L. Vold, K. Stene-Johansen, R. Rimhanen-Finne, M. Kontio, M. Löfdahl, L. Sundqvist, M. Edelstein, T. Jensen, H. T. Vestergaard, T. K. Fischer, K. Mølbak, and S. Ethelberg. 2013. “Ongoing Multi-strain Food-borne Hepatitis A
Outbreak with Frozen Berries as Suspected Vehicle: Four Nordic Countries Affected, October 2012 to April 2013." Euro Surveill. 18 (17): pii=20467. https://www.eurosurveillance.org/ViewArticle.aspx?ArticleId=20467.

Herwaldt, B. L. 2000. "Cyclospora cayetanensis: A Review, Focusing on Outbreaks of Cyclosporiasis in the 1990s." Clin. Infect. Dis. 31 (4): 1040-1057.

Herwaldt, B. L., and M. L. Ackers. 1997. "An Outbreak in 1996 of Cyclosporiasis Associated with Imported Raspberries." N. Engl. J. Med. 336:1548-1556.

Herwaldt, B. L., and M. J. Beach. 1999. "The Return of Cyclospora in 1997: Another Outbreak of Cyclosporiasis in North America Associated with Imported Raspberries. Cyclospora Working Group." Ann. Intern. Med. 130 (3): 210-220.

Hjertqvist, M., A. Johansson, N. Svensson, P. E. Abom, C. Magnusson, M. Olsson, K. O. Hedlund, and Y. Andersson. 2006. "Four Outbreaks of Norovirus Gastroenteritis after Consuming Raspberries, Sweden, June-August 2006.” Euro Surveill. 11 (9): E060907.1.

Ho, A. Y., A. S. Lopez, M. G. Eberhard, R. Levenson, B. S. Finkel, A. J. da Silva, J. M. Roberts, P. A. Orlandi, C. C. Johnson, and B. L. Herwaldt. 2002. "Outbreak of Cyclosporiasis Associated with Imported Raspberries, Philadelphia, Pennsylvania, 2000.” Emerg. Infect. Dis. 8 (8): 783-788.

Hutin, Y. J. F., V. Pool, E. H. Cramer, O. V. Nainan, J. Weth, I. T. Williams, S. T. Goldstein, K. F. Gensheimer, B. P. Bell, C. N. Shapiro, M. J. Alter, and H. S. Margolis. 1999. "A Multistate, Foodborne Outbreak of Hepatitis A.” New Eng. J. Med. 340 (8): 595-602.

Katz, D., S. Kumar, J. Malecki, M. Lowdermilk, E. H. Koumans, and R. Hopkins. 1999. "Cyclosporiasis Associated with Imported Raspberries, Florida, 1996.” Public Health Rep. 114 (5): 427-438.

Korsager, B., S. Hede, H. Boggild, B. E. Bottiger, and K. Molbak. 2005. "Two Outbreaks of Norovirus Infections Associated with the Consumption of Imported Frozen Raspberries, Denmark, May-June 2005." Euro Surveill. 10 (6): E050623.1.

Koumans, E. H., D. J. Katz, J. M. Malecki, S. Kumar, S. P. Wahlquist, M. J. Arrowood, A. W. Hightower, and B. L. Herwaldt. 1998. "An Outbreak of Cyclosporiasis in Florida in 1995: A Harbinger of Multistate Outbreaks in 1996 and 1997." Am. J. Trop. Med. Hyg. 59 (2): 235-242. 
Luna, R. E., and R. Mody. 2010. "Non-O157 Shiga ToxinProducing E. coli (STEC) Outbreaks, United States." CDC Memo to Record. Accessed April 2013. https://blogs.cdc. gov/publichealthmatters/files/2010/05/nono157stec_ obs_052110.pdf.

Marler-Clark, LLP. 2012. "Foodborne Illness Outbreak Database.” Accessed April 2013. http://outbreakdatabase. com/details/pomeberry-blend-frozen-berries2012/?outbreak=berries\&vehicle=berries.

Maunula, L., M. Roivainen, M. Keranen, S. Makela, K. Soderberg, M. Summa, C. H. von Bonsdorff, M. Lappalainen, T. Korhonen, M. Kuusi, and T. Niskanen. 2009. "Detection of Human Norovirus from Frozen Raspberries in a Cluster of Gastroenteritis Outbreaks." Euro Surveill. 14 (49): pii:19435.

Miller, B. D., C. E. Rigdon, T. J. Robinson, C. Hedberg, and K. E. Smith. 2013. "Use of Global Trade Item Numbers in the Investigation of a Salmonella Newport Outbreak Associated with Blueberries in Minnesota, 2010." J. Food Prot. 76 (5): 762-769.

Murrow, L. B., P. Blake, and L. Kreckman. 2002. "Outbreak of Cyclosporiasis in Fulton County, Georgia." Georgia Epidemiol. Rep. 18 (1): 1-2.

Niu, M. T., L. B. Polish, B. H. Robertson, B. K. Kanna, B. A. Woodruff, C. N. Shapiro, M. A. Miller, J. D. Smith, J. K. Gedrose, M. J. Alter, and H. S. Margolis. 1992. "Multistate Outbreak of Hepatitis A Associated with Frozen Strawberries." J. Infect. Dis. 166 (3): 518-524.

Pönkä, A., L. Maunula, C. H. von Bonsdorff, and O. Lyytikäinen. 1999a. "Outbreak of Calicivirus Gastroenteritis Associated with Eating Frozen Raspberries.” Euro. Surveill. 4 (6): 66-69.

Pönkä, A., L. Maunula, C. H. von Bonsdorff, and O. Lyytikäinen. 1999b. "An Outbreak of Calicivirus Associated with Consumption of Frozen Raspberries." Epidemiol. Infect. 123 (3): 469-474.

Oregon Public Health. 2011. "Fresh Strawberries from Washington County Farm Implicated in E. coli O157 outbreak in NW Oregon." Accessed September 2013. https://web.archive.org/web/20121012143345/http://www. oregon.gov/OHA/news/2011/2011-0808.pdf.
Ramsay, C. N., and P. A. Upton. 1989. "Hepatitis A and Frozen Raspberries." Lancet 1 (8628): 43-44.

Reid, T. M. S., and H. G. Robinson. 1987. "Frozen Raspberries and Hepatitis A." Epidemiol. Infect. 98 (1): 109-112.

Rothschild, M. 2012. "BC Issues Warning about Pomeberry Frozen Berries.” Food Safety News. Accessed April 2013. https://www.foodsafetynews.com/2012/04/ bc-issues-warning-about-pomeberry-frozen-berries/.

Sarvikivi, E., M. Roivainen, L. Maunula, T. Niskanen, T. Korhonen, M. Lappalainen, and M. Kuusi. 2012. "Multiple Norovirus Outbreaks Linked to Imported Frozen Raspberries." Epidemiol. Infect. 140 (2): 260-267.

Terry, L. 2011. "Oregon Confirms Deer Droppings Caused E. coli Outbreak Tied to Strawberries." The Oregonian. Accessed September 2013. http://www.oregonlive.com/ washingtoncounty/index.ssf/2011/08/oregon_confirms_deer_droppings.html. 


\begin{tabular}{|c|c|c|c|c|c|c|c|c|c|c|c|c|c|c|}
\hline 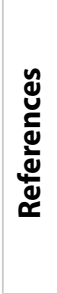 & 㐫 & 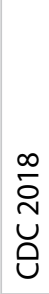 & $\begin{array}{l}\text { O̊ } \\
\text { O } \\
\text { ல் }\end{array}$ & 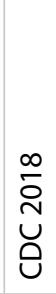 & 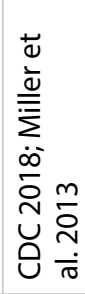 & 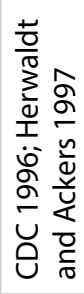 & 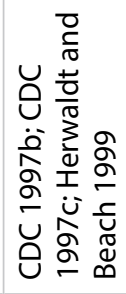 & 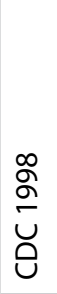 & 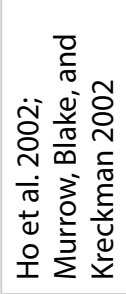 & 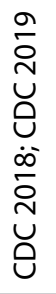 & 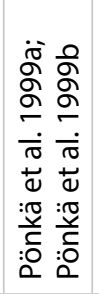 & 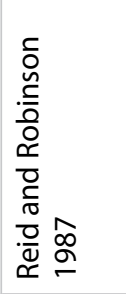 & 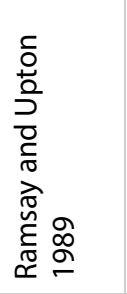 & 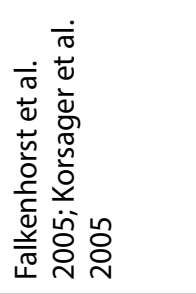 \\
\hline 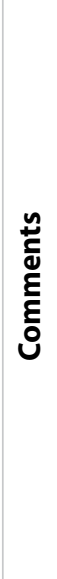 & 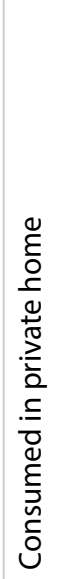 & & 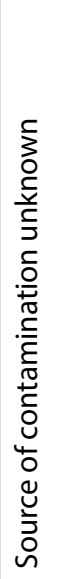 & 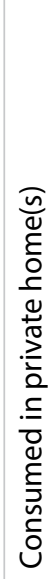 & 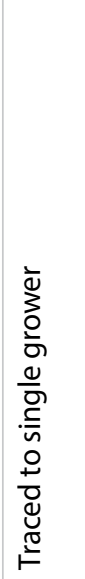 & 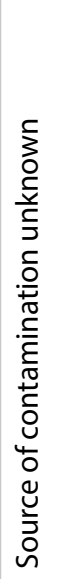 & 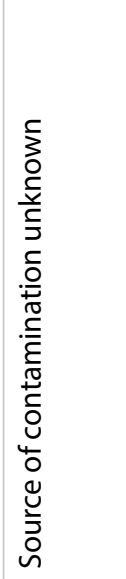 & 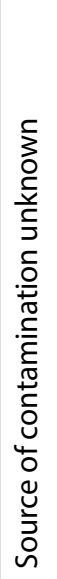 & 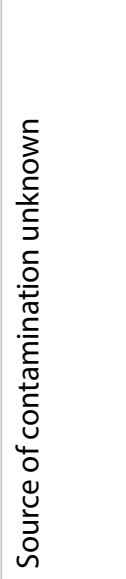 & 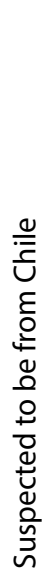 & 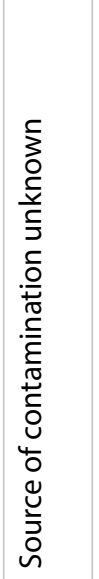 & 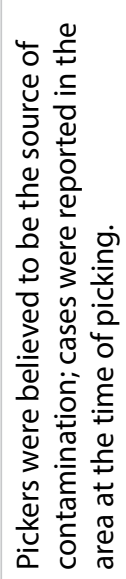 & 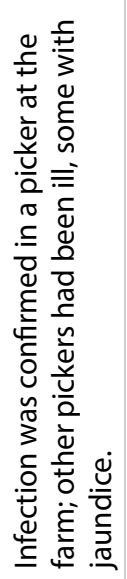 & 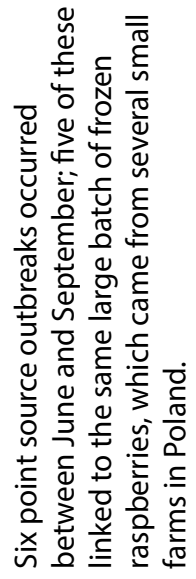 \\
\hline
\end{tabular}
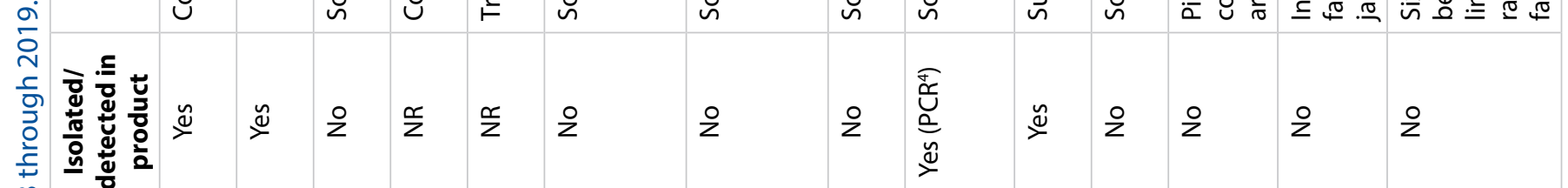

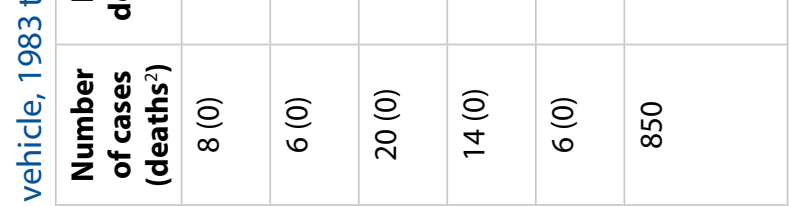
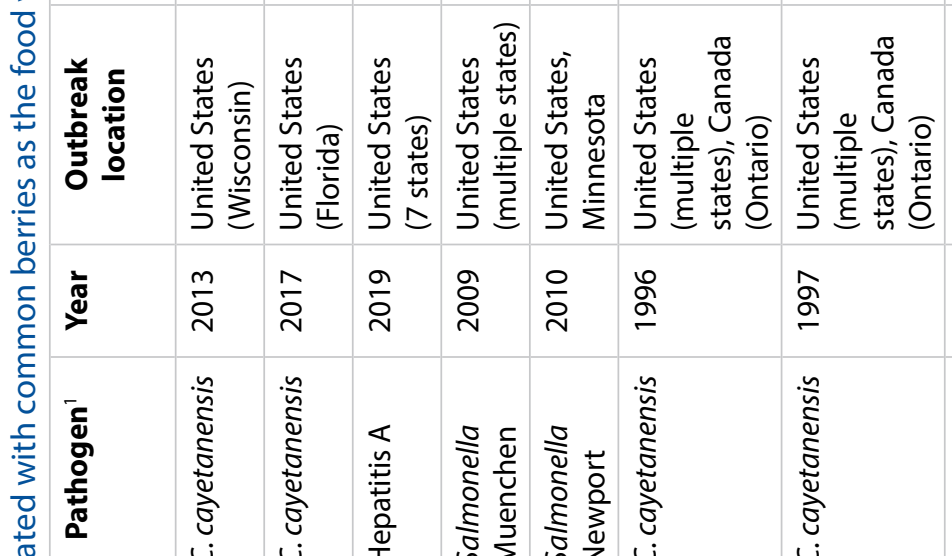

. $\frac{\pi}{\square}$

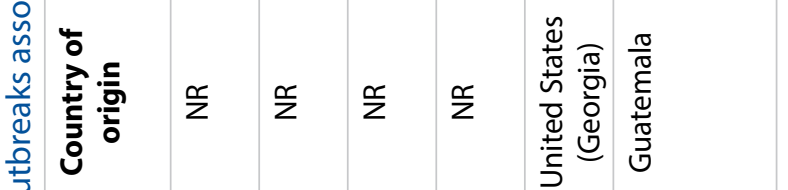

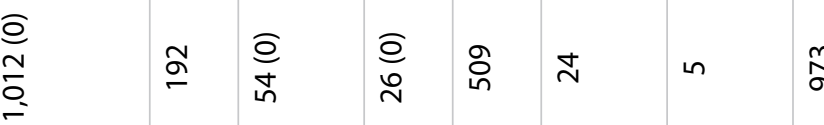

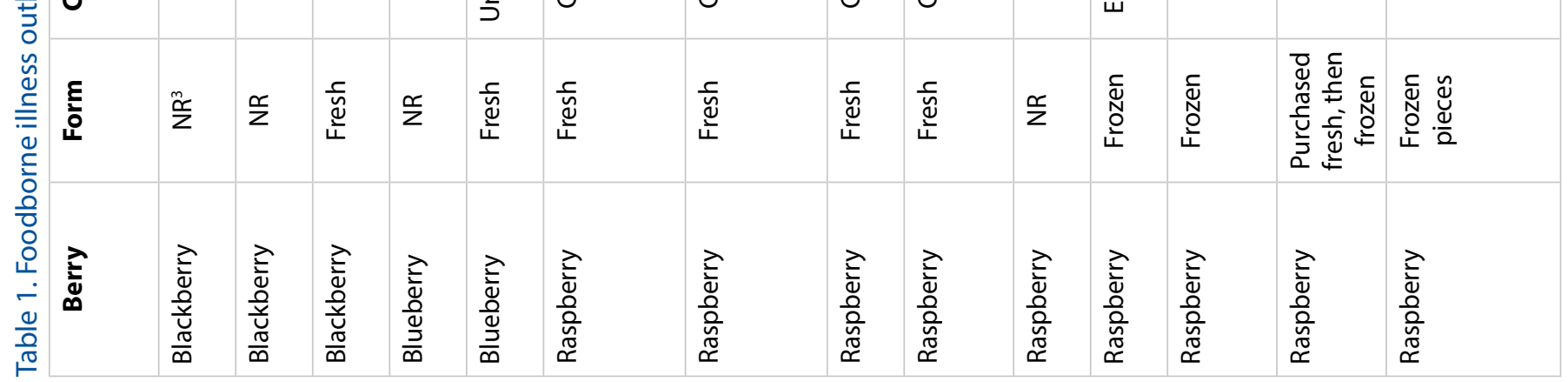




\begin{tabular}{|c|c|c|c|c|c|c|c|}
\hline 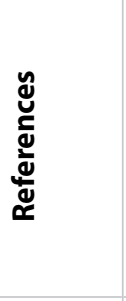 & 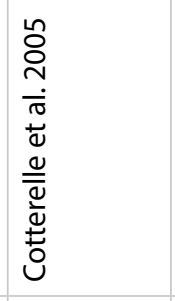 & 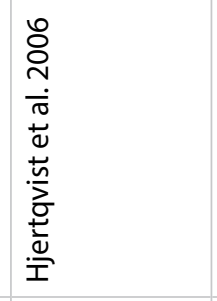 & 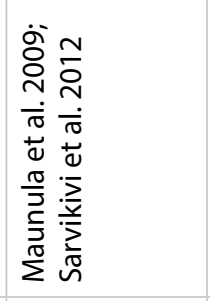 & 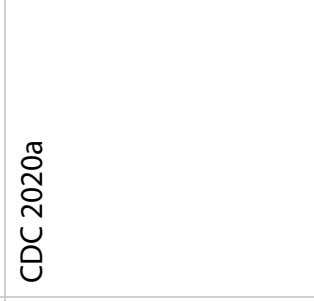 & 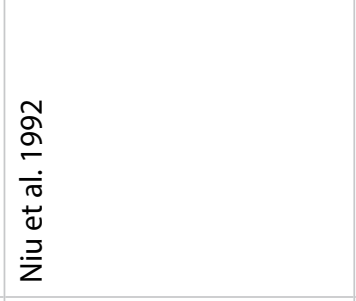 & 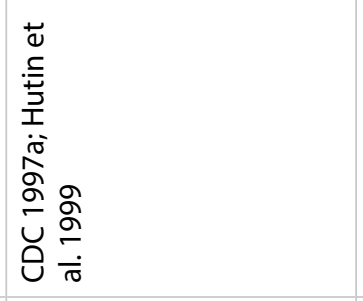 & 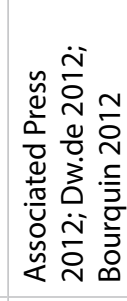 \\
\hline 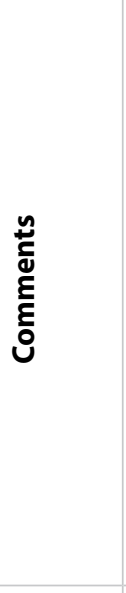 & 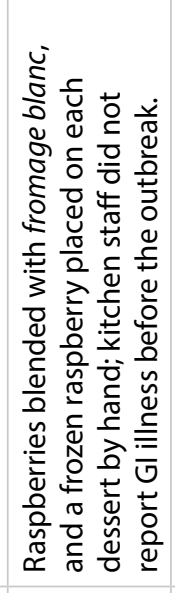 & 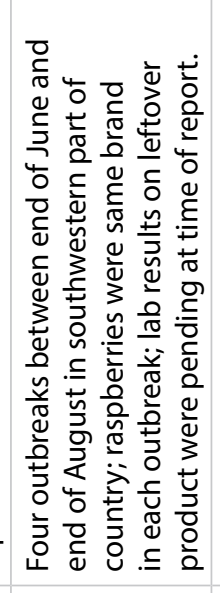 & 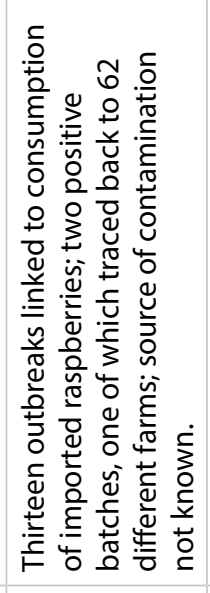 & 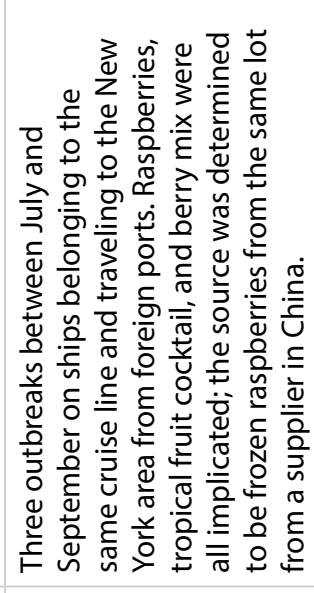 & 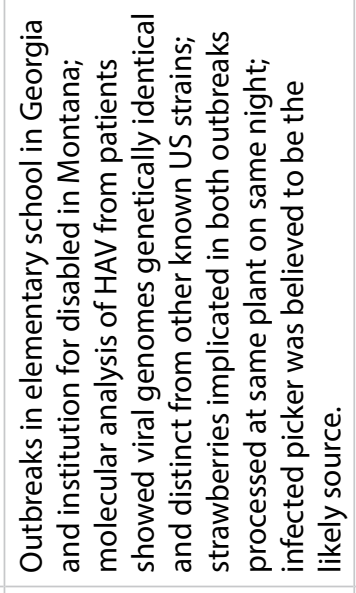 & 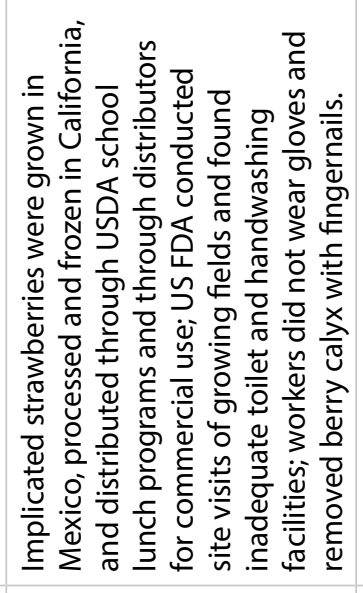 & 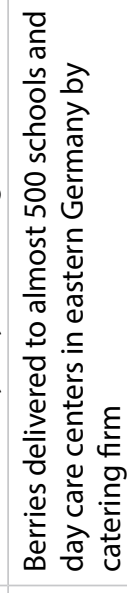 \\
\hline 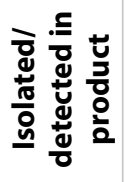 & 울 & 受 & $\stackrel{\check{\nu}}{\check{\nu}}$ & $\stackrel{\mathscr{y}}{=}$ & zo & ㅇ & 愛 \\
\hline 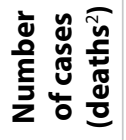 & $\stackrel{n}{\wedge}$ & $\stackrel{m}{f}$ & ৪ి & $\stackrel{m}{\tilde{m}}$ & $\stackrel{\infty}{\sim}$ & $\stackrel{\mathbb{Z}}{\sim}$ & 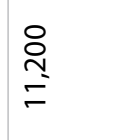 \\
\hline 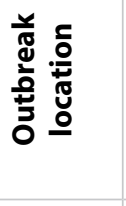 & 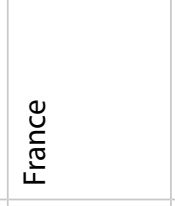 & $\begin{array}{l}\bar{v} \\
\frac{d}{d} \\
\sum_{n}^{\prime}\end{array}$ & 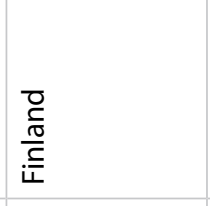 & 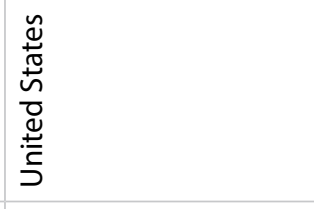 & 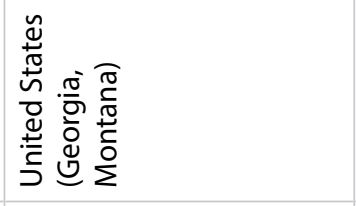 & 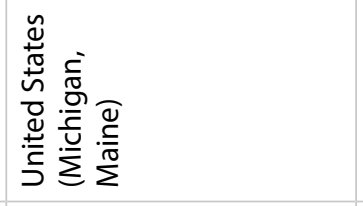 & 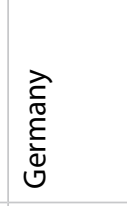 \\
\hline 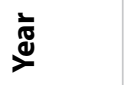 & 哭 & ¿্ণ & ठ্ণ & $\frac{\mathfrak{n}}{2}$ & ᄋ & مे & $\stackrel{\sim}{\grave{N}}$ \\
\hline 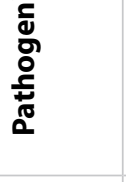 & $\begin{array}{l}\frac{n}{2} \\
\frac{0}{2} \\
\frac{0}{2} \\
z\end{array}$ & $\begin{array}{l}\frac{n}{2} \\
\frac{2}{2} \\
\frac{0}{2} \\
z\end{array}$ & $\frac{n}{\frac{n}{2}}$ & $\frac{n}{\frac{n}{2}}$ & 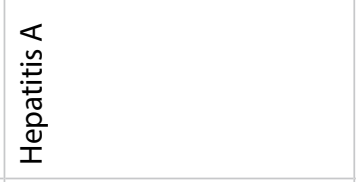 & 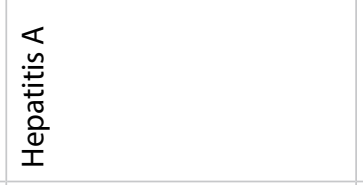 & $\begin{array}{l}\frac{n}{2} \\
\frac{2}{2} \\
\frac{0}{2} \\
2\end{array}$ \\
\hline 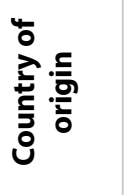 & $\frac{\mathscr{c}}{z}$ & 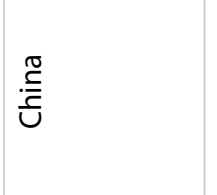 & 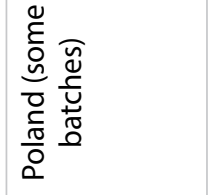 & : & 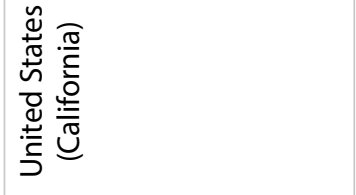 & $\frac{\stackrel{\vartheta}{x}}{\sum^{\frac{U}{2}}}$ & 胥 \\
\hline ह & 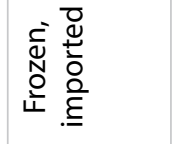 & 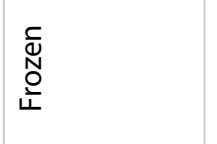 & $\begin{array}{l}\stackrel{\complement}{N} \\
\text { 인 } \\
\text { ㄴ. }\end{array}$ & 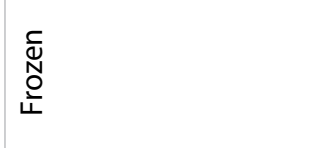 & 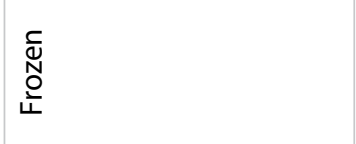 & 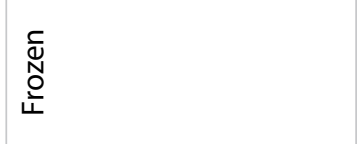 & 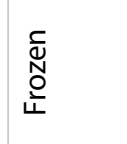 \\
\hline 文 & $\begin{array}{l}\text { ते } \\
\frac{0}{0} \\
\frac{0}{0} \\
\overline{0} \\
\check{c}\end{array}$ & $\begin{array}{l}\text { ते } \\
\frac{0}{0} \\
\frac{0}{0} \\
\overline{0} \\
\check{x}\end{array}$ & 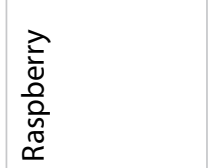 & 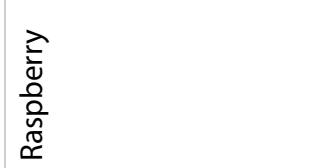 & 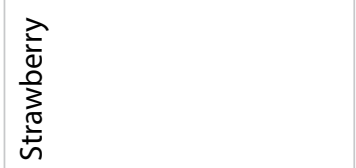 & 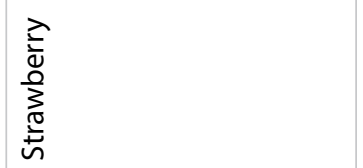 & 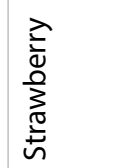 \\
\hline
\end{tabular}




\begin{tabular}{|c|c|c|c|c|c|c|c|c|c|c|c|c|c|}
\hline 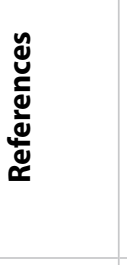 & 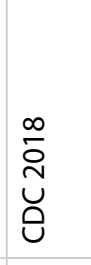 & \begin{tabular}{l}
$\infty$ \\
$\grave{D}$ \\
\hdashline
\end{tabular} & 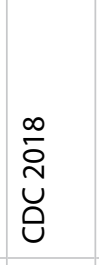 & $\begin{array}{l}\infty \\
\grave{\nu} \\
\check{U}\end{array}$ & 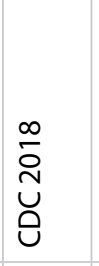 & 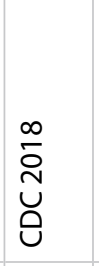 & 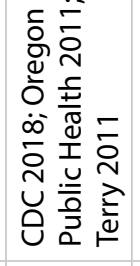 & 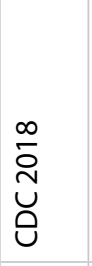 & 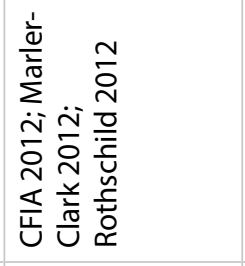 & $\frac{\infty}{\grave{D}}$ & 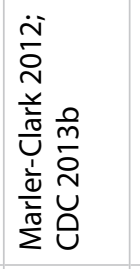 & 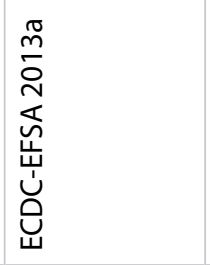 & 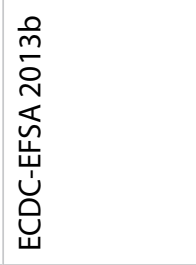 \\
\hline 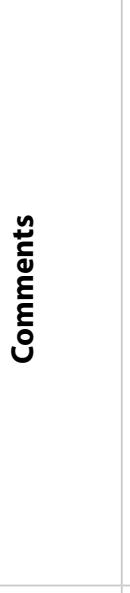 & & & & 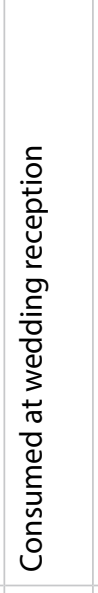 & 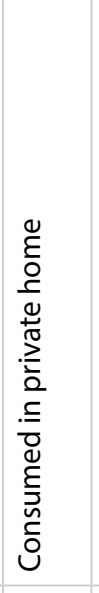 & 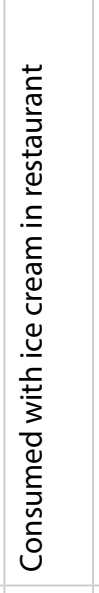 & 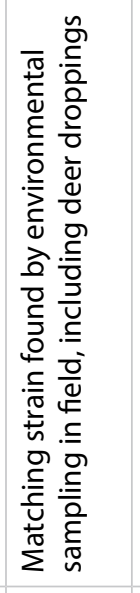 & 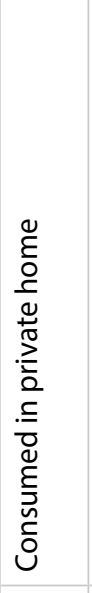 & 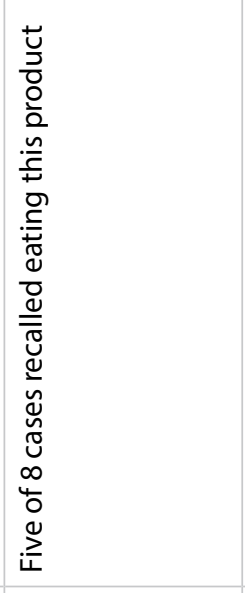 & 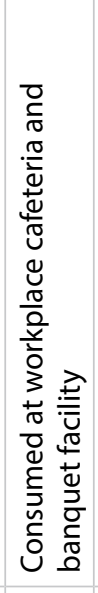 & 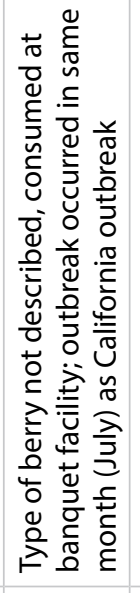 & 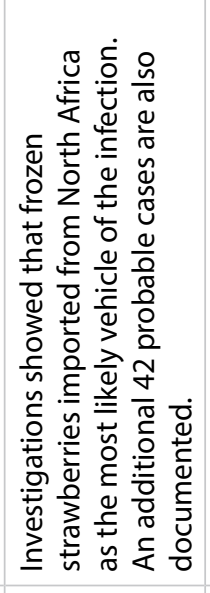 & 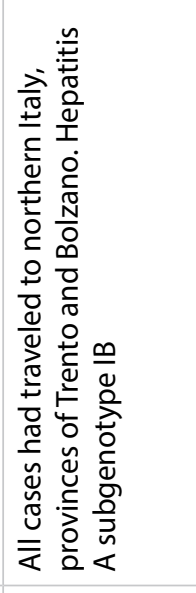 \\
\hline 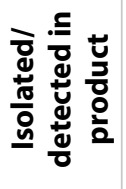 & $\stackrel{\oiiint}{\rightleftharpoons}$ & $\stackrel{\tilde{\nu}}{\check{\nu}}$ & $\stackrel{\tilde{u}}{\succ}$ & $\stackrel{\frac{\Upsilon}{z}}{2}$ & $\stackrel{\frac{N}{z}}{ }$ & $\frac{\mathscr{c}}{z}$ & 受 & $\stackrel{\tilde{\nu}}{\nu}$ & $\stackrel{\frac{N}{z}}{ }$ & $\frac{\mathscr{c}}{z}$ & $\stackrel{\frac{N}{z}}{ }$ & ㄹ & 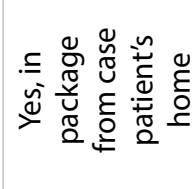 \\
\hline 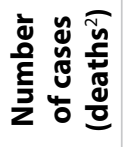 & શิ & $\infty$ & $\stackrel{m}{-}$ & $\begin{array}{l}\text { o } \\
\text { o }\end{array}$ & 흐 & $\stackrel{\underline{a}}{\mathfrak{c}}$ & $\begin{array}{l}\underset{\mathfrak{d}}{n} \\
\underline{n}\end{array}$ & $\stackrel{\bar{a}}{\sim}$ & $\infty$ & $\begin{array}{l}\overline{0} \\
\text { on }\end{array}$ & $\frac{\bar{a}}{m}$ & $\mathbb{Z}$ & $\stackrel{n}{n}$ \\
\hline 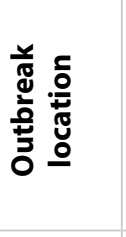 & 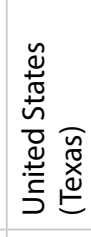 & 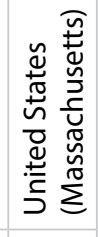 & 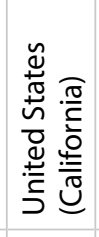 & 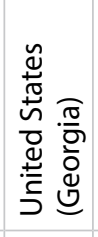 & 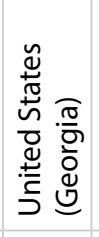 & 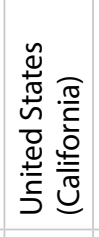 & 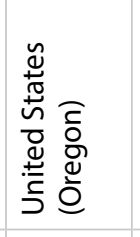 & 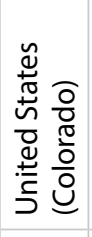 & 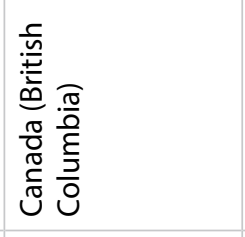 & 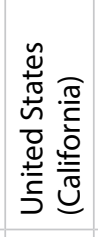 & 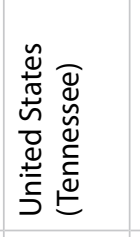 & 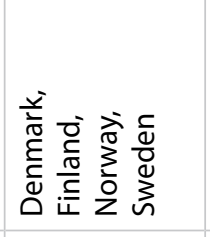 & 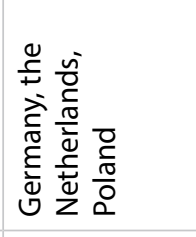 \\
\hline 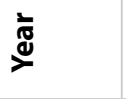 & $\stackrel{\infty}{\circ}$ & ঠ্ণ & ڤి & 苂 & ڤ્ণ & ڤ్̀ે & $\overline{\bar{\nu}}$ & $\frac{m}{\grave{N}}$ & ָั & ঃ̊̀ & $\stackrel{\infty}{\circ}$ & $\begin{array}{l}\stackrel{1}{m} \\
\stackrel{m}{i} \\
\stackrel{N}{N}\end{array}$ & $\stackrel{m}{\grave{n}}$ \\
\hline 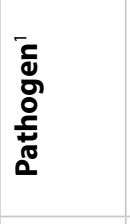 & 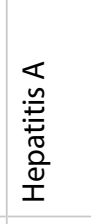 & 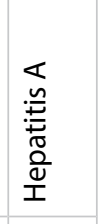 & 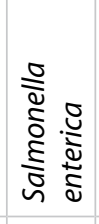 & $\begin{array}{l}\stackrel{n}{2} \\
\frac{2}{2} \\
\frac{0}{2}\end{array}$ & $\frac{n}{\frac{n}{2}}$ & $\begin{array}{l}\frac{n}{2} \\
\frac{n}{2} \\
\frac{0}{2} \\
\frac{0}{2}\end{array}$ & 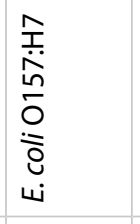 & 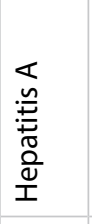 & 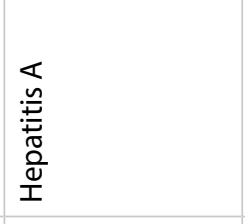 & 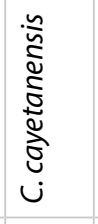 & 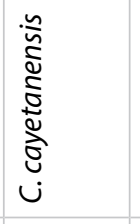 & 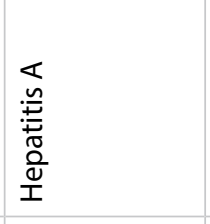 & 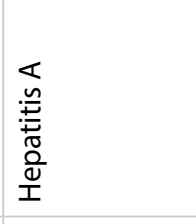 \\
\hline 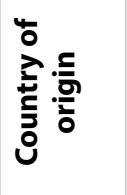 & $\stackrel{\Upsilon}{z}$ & $\frac{\mathscr{c}}{z}$ & $\frac{\mathscr{c}}{z}$ & 号 & 愛 & 号 & 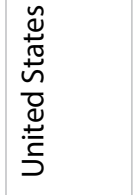 & $\frac{\mathscr{c}}{z}$ & 愛 & $\frac{\mathscr{c}}{z}$ & $\stackrel{\frac{c}{z}}{z}$ & $\frac{\mathscr{c}}{z}$ & $\stackrel{\mathscr{c}}{z}$ \\
\hline ్ㅗํ & 受 & $\frac{\mathscr{c}}{z}$ & $\frac{\mathscr{c}}{z}$ & 紜 & $\stackrel{\mathscr{c}}{z}$ & 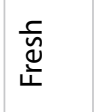 & 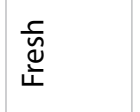 & $\frac{\mathfrak{c}}{z}$ & $\begin{array}{l}\bar{N} \\
\text { N } \\
\text { 니 }\end{array}$ & 兴 & 寽 & 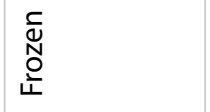 & $\begin{array}{l}\bar{N} \\
\text { N } \\
\frac{0}{4}\end{array}$ \\
\hline 竞 & 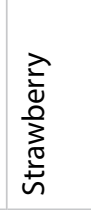 & 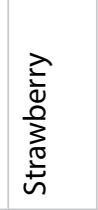 & $\begin{array}{l}\text { 离 } \\
\frac{0}{3} \\
\text { 离 } \\
\text { w }\end{array}$ & 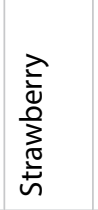 & 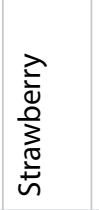 & 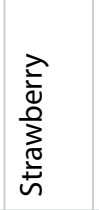 & 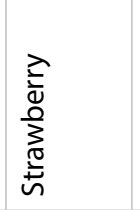 & 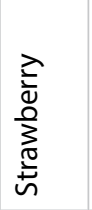 & 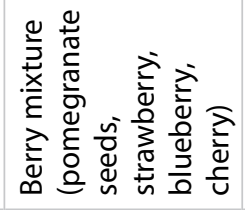 & 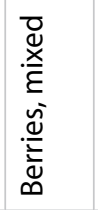 & 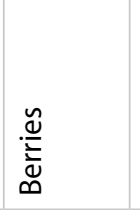 & 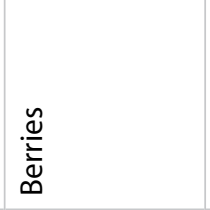 & 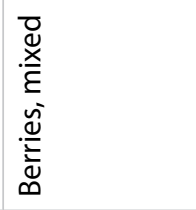 \\
\hline
\end{tabular}




\begin{tabular}{|c|c|c|c|c|c|c|}
\hline 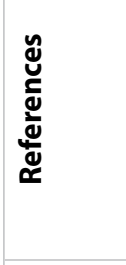 & 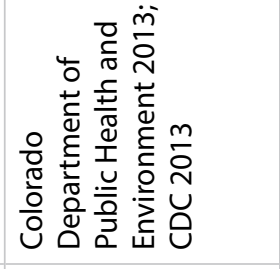 & 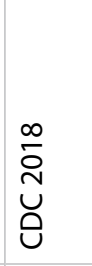 & 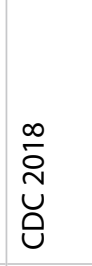 & $\frac{\infty}{\grave{N}}$ & 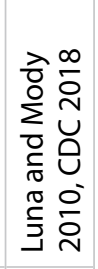 & \\
\hline 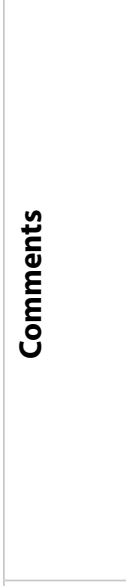 & 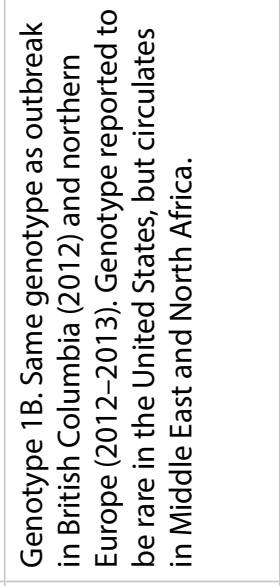 & 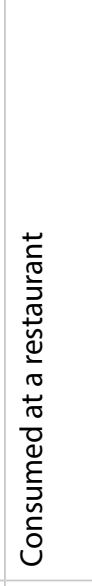 & 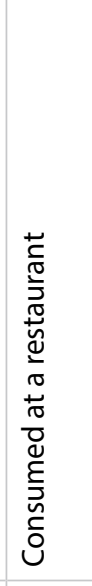 & 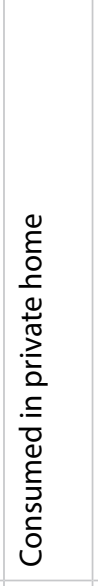 & & 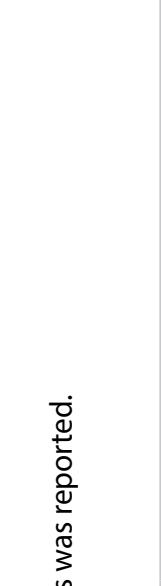 \\
\hline 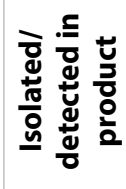 & 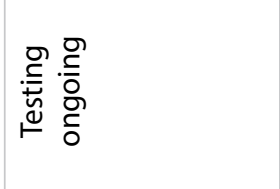 & $\stackrel{\Perp}{\nu}$ & $\stackrel{\check{\nu}}{\check{\nu}}$ & $\stackrel{\tilde{\nu}}{\nu}$ & 哭 & 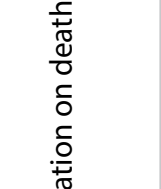 \\
\hline 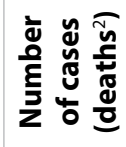 & i & ઠ & $\begin{array}{l}\overline{0} \\
\text { a } \\
-\end{array}$ & $\underset{\infty}{\bar{\varrho}}$ & $\underset{\mathrm{o}}{\overline{\mathrm{O}}}$ & 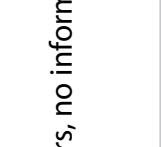 \\
\hline 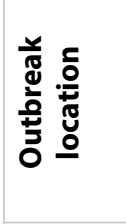 & 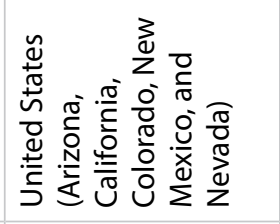 & 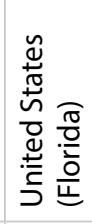 & 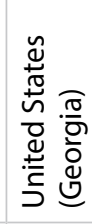 & 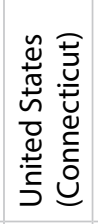 & 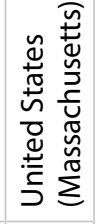 & 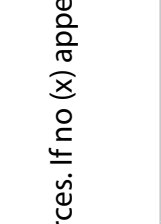 \\
\hline 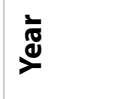 & $\stackrel{m}{i}$ & જે & ঠ & ஓे & 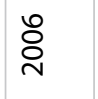 & $\begin{array}{l}\overrightarrow{0} \\
\stackrel{n}{0} \\
\stackrel{\Xi}{\Xi}\end{array}$ \\
\hline 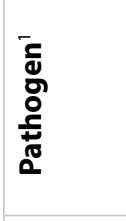 & 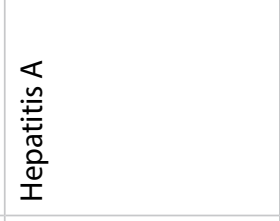 & 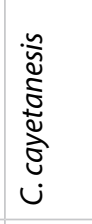 & 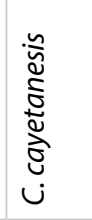 & 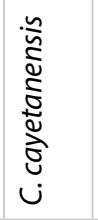 & $\begin{array}{l}\stackrel{0}{0} \\
\stackrel{0}{0} \\
\text { u }\end{array}$ & 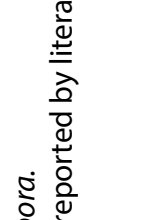 \\
\hline 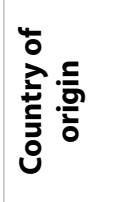 & 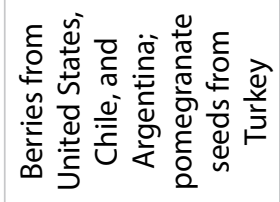 & $\stackrel{\Upsilon}{z}$ & 愛 & 愛 & $\frac{\mathscr{c}}{z}$ & 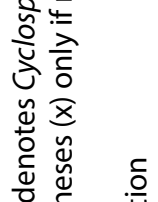 \\
\hline ह & 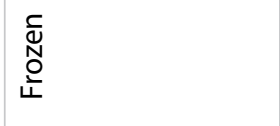 & 愛 & 愛 & 愛 & 愛 & 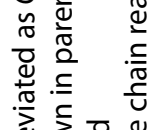 \\
\hline गิ & 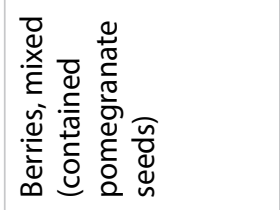 & 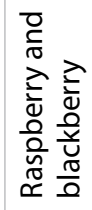 & 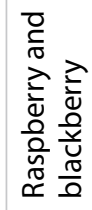 & 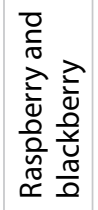 & 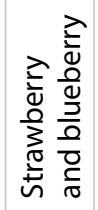 & 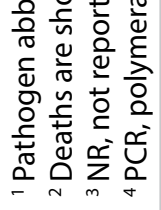 \\
\hline
\end{tabular}




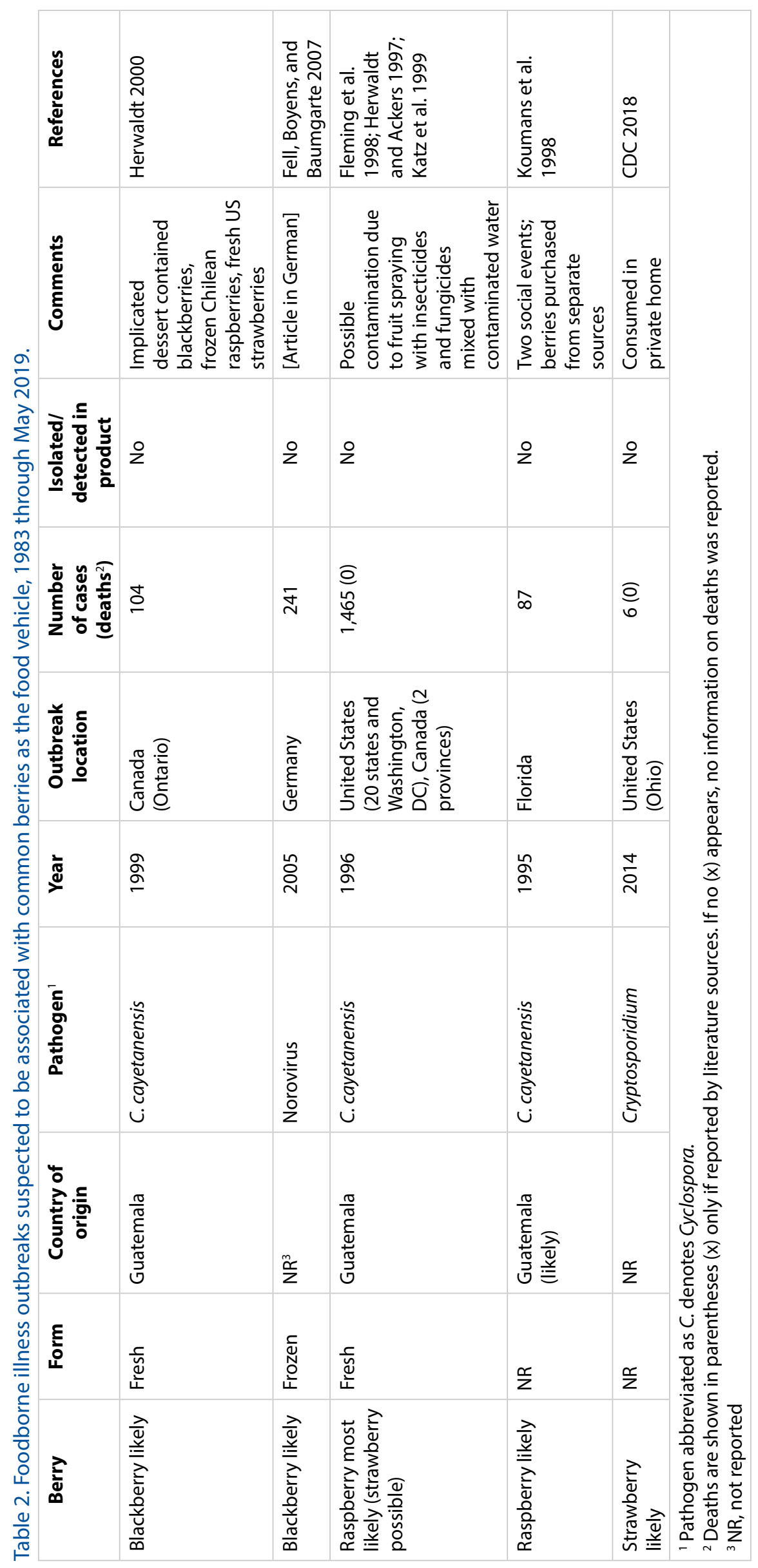

